\title{
LENGTH ESTIMATES FOR HOLOMORPHIC FUNCTIONS
}

\author{
SHINJI YAMASHITA
}

\begin{abstract}
Let $f(z)=a_{0}+\sum_{k=n}^{\infty} a_{k} z^{k}(n>1)$ be holomorphic in $U:|z|<1$. MacGregor [1, Theorem 2] proved that if $l(r, f)$ is the length of the outer boundary of the image $D(r, f)$ of the disk: $|z|<r$ by $f$, then $2 \pi r^{n}\left|a_{n}\right|<l(r, f)$ for $0<r<1$. We introduce the notion of the exact outer boundary $C^{\sharp}(r, f)$ of $D(r, f)$ and prove that $2 \pi r^{n}\left|a_{n}\right|<l^{\sharp}(r, f)<l(r, f)$ for $0<r<1$, where $l^{\sharp}(r, f)$ is the length of $C^{\sharp}(r, f)$. We shall make use of the estimate to obtain a criterion for $f$ to be Bloch in $U$.
\end{abstract}

In this note $f$ is always a function nonconstant and holomorphic in the disk $U=\{|z|<1\}$. Let $D(r, f)$ be the image of the disk $\{|z|<r\}$ by $f(0<r<1)$, namely, $D(r, f)$ is the projection of the Riemannian image of $\{|z|<r\}$ by $f$, to the plane: $|z|<\infty$. The boundary $C(r, f)$ of the unbounded component of the complement of $D(r, f)$ is called the outer boundary of $D(r, f)$. If, in the definition of $C(r, f)$, we replace $D(r, f)$ by its closure, then we obtain the boundary $C^{\sharp}(r, f)$, called the exact outer boundary of $D(r, f)$. Let $l(r, f)\left(l^{\sharp}(r, f)\right.$, respectively) be the length of $C(r, f)\left(C^{\sharp}(r, f)\right.$, resp.). Apparently, $l^{\sharp}(r, f) \leqslant l(r, f)$. T. H. MacGregor [1, Theorem 2] proved that, if

$$
f(z)=a_{0}+\sum_{k=n}^{\infty} a_{k} z^{k} \quad(n>1)
$$

is the Taylor expansion in $U$, then

$$
2 \pi r^{n}\left|a_{n}\right|<l(r, f) \quad(0<r<1) .
$$

We shall call this Theorem $\mathbf{M}$. The following theorem improves Theorem $\mathbf{M}$.

THEOREM 1. If (1) holds, then

$$
2 \pi r^{n}\left|a_{n}\right|<l^{\sharp}(r, f) \quad(0<r<1) .
$$

Before the proof we propose an example of $f$ such that $l^{\sharp}(r, f)<l(r, f)$ for an $r$. Consider $g(z)=(c z+2 / \sqrt{3})^{3}$, where $c>1$ is a constant. Then $l^{\sharp}\left(c^{-1}, g\right)<$ $l\left(c^{-1}, g\right)$, in other words, $l\left(c^{-1}, g\right)-l^{\sharp}\left(c^{-1}, g\right)$ is the legth of the "shoreline" of the "bay" of the "island" $D\left(c^{-1}, g\right)$. I am indebted to the referee for this example; my example in the first draft was false.

We remember the isoperimetric theorem.

LEMMA. Of all rectifiable Jordan curves with a given perimeter, the circle has the interior of the maximum area.

Received by the editors October 1, 1979 and, in revised form, February 25, 1980. 1980 Mathematics Subject Classification. Primary 30C99, 30D45; Secondary 30C80. 
Since $C^{\sharp}(r, f)$ is a Jordan curve consisting of a finite number of analytic arcs, it is rectifiable. Let $a(r, f)$ be the area of $D(r, f)$, and let $A$ be the area of the Jordan domain $\mathscr{D}$ with the boundary $C^{\sharp}(r, f)$. Then $a(r, f)<A$. It follows from the Lemma that

$$
A \leqslant \pi\left[l^{\sharp}(r, f) /(2 \pi)\right]^{2} .
$$

On the other hand [1, Theorem 1],

$$
\pi r^{2 n}\left|a_{n}\right|^{2}<a(r, f) \text {. }
$$

The inequality (3) now follows from (4) and (5).

The referee proposed the following alternative proof. Let a univalent holomorphic function $h$ map $U$ onto $\mathscr{D}$, such that $h(0)=f(0)$. Set $c_{1}=h^{\prime}(0)$. Then Theorem M, together with the limiting process, yields that

$$
2 \pi\left|c_{1}\right| \leqslant \lim _{s \rightarrow 1-0} l^{\sharp}(s, h)=l^{\sharp}(r, f) .
$$

Since $g(z) \equiv f(r z)$ is subordinate to $h$, the same argument as in the proof of Theorem $M$ in [1] is available, so that

$$
r^{n}\left|a_{n}\right|=\left|g^{(n)}(0)\right| / n !<\left|c_{1}\right| \text {. }
$$

Therefore (3) is established.

Let

$$
\Delta(z, r)=\{w \in U ;|w-z| /|1-\bar{z} w|<r\}
$$

be the non-Euclidean hyperbolic disk of the non-Euclidean center $z \in U$ and the non-Euclidean radius $\frac{1}{2} \log [(1+r) /(1-r)](0<r<1)$. Let $D(r, f, z)$ be the image of $\Delta(z, r)$ by $f$, and let $l^{\sharp}(r, f, z)$ be the length of the exact outer boundary of $D(r, f, z)$, that is, $l^{\sharp}(r, f, z)=l^{\sharp}(r, g)$ for the composed function

$$
g(w)=f((w+z) /(1+\bar{z} w))
$$

of a variable $w \in U$.

We consider a localization of Theorem 1 in

THEOREM 2. Let $n \equiv n(z, f) \geqslant 1$ be the first number such that $f^{(n)}(z) \neq 0$ at $z \in U$. Then

$$
2 \pi r^{n}\left(1-|z|^{2}\right)^{n}\left|f^{(n)}(z)\right| / n !<l^{\sharp}(r, f, z)
$$

for all $r, 0<r<1$.

Since $f$ is nonconstant, $n(z, f)$ always exists.

For the proof of (7) we apply (3) to $g$ of (6). Since

$$
g^{(n)}(0) / n !=\left(1-|z|^{2}\right)^{n} f^{(n)}(z) / n !
$$

we obtain (7).

The case $n=1$ is of particular interest. If $f^{\prime}(z) \neq 0$, then

$$
\left(1-|z|^{2}\right)\left|f^{\prime}(z)\right|<l^{\sharp}(r, f, z) /(2 \pi r),
$$

while if $f^{\prime}(z)=0$, the estimate (8) is trivial. Therefore, if there exists $r, 0<r<1$, such that

$$
\sup _{z \in U} l^{\sharp}(r, f, z)<\infty,
$$


then $f$ is Bloch $([2],[3$, p. 268]) in the sense that

$$
\sup _{z \in U}\left(1-|z|^{2}\right)\left|f^{\prime}(z)\right|<\infty \text {. }
$$

Conversely, if $f$ is Bloch, then (9) holds for each $r, 0<r<1$. In effect, let $K$ be the supremum of (10) and let $\mathcal{L}(r, f, z)$ be the length of the boundary of the Riemannian image of $\Delta(z, r)$ by $f$. Then

$$
\begin{aligned}
\mathcal{L}(r, f, z) & =\int_{\partial \Delta(z, r)}\left|f^{\prime}(w)\right||d w| \\
& \leqslant K \int_{|w|=r}\left(1-|w|^{2}\right)^{-1}|d w|=2 \pi K r\left(1-r^{2}\right)^{-1} .
\end{aligned}
$$

Actually, $\left(1-|w|^{2}\right)^{-1}|d w|$ is invariant for the non-Euclidean transformations. Thus, (9) is a consequence of $l^{\sharp}(r, f, z)<\mathcal{L}(r, f, z)$.

By the similar argument we also have a localization of [1, Theorem 1], which we leave as an exercise.

I wish to express my cordial thanks to the referee for his corrections and criticisms. Without his help, the present work would surely have been aborted.

\section{REFERENCES}

1. Thomas H. MacGregor, Length and area estimates for analytic functions, Michigan Math. J. 11 (1964), 317-320.

2. Christian Pommerenke, On Bloch functions, J. London Math. Soc. (2) 2 (1970), 689-695.

3. , Univalent functions, Vandenhoeck \& Ruprecht, Göttingen, 1975.

Department of Mathematics, Tokyo Metropolttan University, Fukazawa, Setagaya, Tokyo 158, JAPAN 